\title{
Medium influence of the nucleon nucleon cross section on the fragmentation
}

\author{
Jian-Ye Liu ()$^{1,2,3,4}$, Yong-Zhong Xing ()$^{1,2}$, Wen-Jun Guo ()$^{3}$ \\ ${ }^{1}$ Institute for the theory of modern physics, Tianshui Normal University, Gansu, \\ Tianshui 741000, P. R. China \\ ${ }^{2}$ Center of Theoretical Nuclear Physics, National Laboratory of Heavy Ion Accelerator \\ Lanzhou 730000, P.R. China \\ ${ }^{3}$ Institute of Modern Physics, Chinese Academy of Sciences, P.O.Box 31 \\ Lanzhou 730000, P.R. China \\ ${ }^{4}$ CCAST(Word Lab.),P.O.Box 8730,Beijing 100080 \\ Lanzhou 730000, P.R. China \\ Based on an isospin dependent quantum molecular dynamics model we stud- \\ ied the influence of a medium correction of an isospin dependent nucleon nu- \\ cleon cross section on the fragmentation at the intermediate energy heavy ion \\ collisions. We found that the medium correction from an isospin dependent \\ nucleon nucleon cross section increases the dependence of the fragmentation \\ on the isospin effect of in-medium nucleon nucleon cross section, at the same \\ time, the momentum dependent interaction (MDI) produces also an important \\ role for enhancing the influence of the medium correction on the isospin effect \\ of two-body collisions in the fragmentation process.
}

PACS number(s): $25 \cdot 70 \cdot \mathrm{pq}$

Correspond auther: E-mail address: Liujy@ns.lzb.ac.cn

Phone number:86-0931-4969318(O),8272215(H). Fax number:86-0931-4969201

The rapid progress in producing energetic radioactive beams has offered an excellent opportunity to investigate various isospin effects in the dynamics of nuclear reaction $[1-5]$. This study has made it possible to obtain crucial information about the equation of state of isospin asymmetric nuclear matter and the isospin dependent in-medium nucleon nucleon cross section. Recently, several interesting isospin effects in heavy ion collisions have been explored both experimentally $[6-16,36]$ and theoretically $[17-29,37,38]$. For a recent review, see, e.g., Ref. [1]. However, two essential ingredients in heavy-ion collision dynamics, i.e., the symmetry potential of the mean field and the isospin dependent in-medium nucleon nucleon cross section are still not well determined. Based on an isospin dependent quantum molecular dynamics model(IQMD) we studied the underlying influence of the isospin effect of 
an in-medium nucleon nucleon cross section on the fragmentation and the dissipation in intermediate energy heavy ion collisions. We found the pronounced isospin effects in the nuclear stopping, the multiplicity of intermediate mass fragments and the number of nucleon emissions due to mainly the isospin dependent in-medium nucleon nucleon cross section $[35,36,37]$. Furthermore, we investigate the influences of the medium correction of an isospin dependent nucleon nucleon cross section on the isospin effects of two-body collision in fragmentation at intermediate energy heavy ion collisions. The calculated results show that the medium correction of an isospin dependent nucleon nucleon cross section enhances the dependence of the multiplicity of intermediate mass fragments $N_{i m f}$ on the isospin effect of in-medium nucleon nucleon cross sections, while MDI produces an important role for inducing above effects in the fragmentation process. As well know that quantum molecular dynamics (QMD) model [30,31] contains two dynamical ingredients, the density dependent mean field and the in-medium nucleon nucleon cross section. In order to describe the isospin dependence appropriately, the QMD model should be modified properly. Considering the isospin effects in mean field,two-body collision and Pauli blocking as shown in Ref.[38] we have made important modifications in QMD to obtain an isospin dependent quantum molecular dynamics (IQMD) [1,34]. The initial density distributions of the colliding nuclei in IQMD are obtained from the calculations of the Skyrme-Hatree-Fock with parameter set SKM* [32]. The parameters of interaction potential was obtained by comparing the calculated results with the experimental data of the ground state properties of coliiding nuclei. The interaction potential is

$$
U(\rho)=U^{S k y}+U^{c}+U^{s y m}+U^{Y u k}+U^{M D I}+U^{\text {Pauli }},
$$

where $U^{S k y}, U^{c}, U^{Y u k}, U^{M D I}$ and $U^{P a u l i}$ are Skyrme potential , Coulomb potential, the Yukawa potential, the momentum dependent interaction and Pauli potentials. We used the following two different forms of the symmetry potentials $[1,2]$

$$
\begin{gathered}
U_{1}^{\text {sym }}=c u \delta \tau_{z} \\
U_{2}^{\text {sym }}= \pm c u^{1 / 2} \delta-\frac{1}{4} c u^{1 / 2} \delta^{2} \\
U_{0}^{\text {sym }}=0.0
\end{gathered}
$$


where

$$
\tau_{z}= \begin{cases}1 & \text { for neutron } \\ -1 & \text { for proton }\end{cases}
$$

and $\mathrm{c}$ is the strength of the symmetry potential being a value of $32 \mathrm{MeV}$ and $U_{0}^{\text {sym }}$ means without symmetry potential. In this work, $u=\frac{\rho}{\rho_{0}}$ is the reduced density and $\delta=\frac{\rho_{n}-\rho_{p}}{\rho_{n}+\rho_{p}}=\frac{\rho_{n}-\rho_{p}}{\rho}$ is the relative neutron excess. $\rho, \rho_{0}, \rho_{n}$ and $\rho_{p}$ are the total , normal, neutron and proton densities, respectively. The recent studies of collective flow in heavy ion collisions at intermediate energy have indicated a reduction of in-medium nucleon nucleon cross sections. An empirical density dependent nucleon nucleon cross section in medium [26] has been suggested as follows

$$
\sigma_{N N}=\left(1+\alpha \frac{\rho}{\rho_{0}}\right) \sigma_{N N}^{\text {free }}
$$

where $\sigma_{N N}^{\text {free }}$ is the experimental nucleon nucleon cross section [33]. The expression (5) with $\alpha=-0.2$ has been found to reproduce the flow data. The free neutron proton cross section is about a factor of 3 times larger than the free proton proton or the free neutron neutron one below $400 \mathrm{MeV}$, which contributes the main isospin effect from nucleon-nucleon collisions. The main physics ingredients and their numerical realization in the IQMD model can be found in Refs $[1,30,31,34]$.

The isospin effect of the in-medium nucleon nucleon cross section on the observables is defined by the difference between the observable from an isospin dependent nucleon nucleon cross section $\sigma^{i s o}$ and that from an isospin independent nucleon nucleon cross section $\sigma^{\text {noiso }}$ in the medium. Here $\sigma^{i s o}$ is defined as $\sigma_{n p} \geq \sigma_{n n}=\sigma_{p p}$ and $\sigma^{\text {noiso }}$ means $\sigma_{n p}=\sigma_{n n}=\sigma_{p p}$, where $\sigma_{n p}, \sigma_{n n}$ and $\sigma_{p p}$ are the neutron proton, neutron neutron and proton proton cross sections in medium respectively. In order to study the influence played by the medium correction of the isospin dependent nucleon nucleon cross section on the isospin effects of two-body collision in the fragmentation process including the contributions from all of impact parameters to multiplicity of intermediate mass fragments $<N_{i m f}>_{b}$, Fig.1 shows the impact parameter average values of $<N_{i m f}>_{b}$ (from at equilibrium time $200 \mathrm{fm} / \mathrm{c}$ ) as a function of the beam energy for the mass symmetry system ${ }^{76} K_{r}+{ }^{76} K_{r}$ (top panels) and mass asymmetry system ${ }^{112} S_{n}+{ }^{40} C_{a}$ (bottom panels) with the same system mass for the different symmetry potentials $U_{1}^{s y m}, U_{2}^{s y m}$ and $U_{0}^{\text {sym }}$, with the isospinm 
dependent in-medium nucleon nucleon cross section $\sigma^{i s o}$ and the isospin independent one $\sigma^{\text {noiso }}$. Namely, there are $U_{0}^{\text {sym }}+\sigma^{\text {iso }}, U_{1}^{\text {sym }}+\sigma^{\text {iso }}, U_{2}^{\text {sym }}+\sigma^{i s o}, U_{1}^{\text {sym }}+\sigma^{\text {noiso }}$ and $U_{2}^{\text {sym }}+\sigma^{\text {noiso }}$ with $\alpha=-0.2$ (left panels) and $\alpha=0.0$ (right panels) in Fig.1. The multiplicity of the intermediate mass fragments $\left\langle N_{i m f}\right\rangle_{b}$ is defined as the fragments with charge numbers from 3 to 13 . It is clear to see that all of lines with filled symbols are larger than those with open symbols,i.e., all of $\left\langle N_{i m f}\right\rangle_{b}$ with $\sigma^{i s o}$ are larger than those with $\sigma^{\text {noiso }}$ because the collision number with $\sigma^{i s o}$ is larger than that with $\sigma^{\text {noiso }}$.We also found that the gaps between lines with the filled symbols and the open symbols are larger but the variations among lines in each group are smaller. These mean that $\left\langle N_{i m f}>_{b}\right.$ depends sensitively on the isospin effect of in-medium nucleon nucleon cross section and weakly on the symmetry potential. In particular, the gaps between two group lines with $\alpha=-0.2$ in left panels are larger than corresponding those with $\alpha=0.0$ in right panels, i.e., the medium correction of two-body collision enhances the dependence of $\left\langle N_{i m f}\right\rangle_{b}$ to its isospin effect. We also found an important role of the MDI for enhancing the isospin effect correlating with the medium correction of two-body collision on the $\left\langle N_{i m f}\right\rangle_{b}$. Fig.2 shows the time evolusion of $N_{i m f}$ for a symmetry potentials $U_{1}^{\text {sym }}$ at beam energy of $100 \mathrm{MeV} /$ nucleon and impact parameter of $4 \mathrm{fm}$. They are four cases: (1) $\alpha=-0.2+\sigma^{\text {iso }},(2) \alpha=0.0+\sigma^{i s o},(3) \alpha=-0.2+\sigma^{\text {noiso }}$ and (4) $\alpha=0.0+\sigma^{\text {noiso }}$ with MDI in the left window and NOMDI in the right window. It is clear to see that the gap between the solid line and dot line with MDI in the left window are larger than corresponding gap with NOMDI in the right window in the medium $(\alpha=0 .-2)$, i.e., MDI increases the isospin effect of two-body collision on the $N_{i m f}$ in the medium because above gap is produced from the isospin effect of nucleon-nucleon cross section in the medium. Why does the medium correction of nucleon nucleon cross section and MDI enhance the dependence of $N_{i m f}$ on the isospin effect of two-body collision? Physically there are three mechanisms at work here.(1) The average momentum of a particle in medium is higher in a heavy ion collision than in cold nuclear matter at the same density. (2) MDI induces the transporting momentum more effectively from one part of the system to another, in which particles also move with a higher velocity for a given momentum than in free 
space. (3) As well know that the isospin dependent in-medium nucleon nucleon cross section is a sensitive founction of the nuclear density distribution and beam energy as shown in Eg.(5). Fig.3 shows the time evolution of the ratio of nuclear density to normal one, $\frac{\rho}{\rho_{0}}$ for four cases: they are $\rho\left(\sigma^{i s o}, \alpha=-0.2\right), \rho\left(\sigma^{\text {noiso }}, \alpha=-0.2\right)$, $\rho\left(\sigma^{i s o}, \alpha=0.0\right)$ and $\rho\left(\sigma^{\text {noiso }}, \alpha=0.0\right)$ for the reaction ${ }^{76} \mathrm{Kr}+{ }^{76} \mathrm{Kr}$ and symmetry potential $U_{1}^{\text {sym }}$ at $\mathrm{E}=150 \mathrm{MeV} /$ nucleon and $\mathrm{b}=4.0 \mathrm{fm}$. From the values of peak for $\frac{\rho}{\rho_{0}}$ in the insert in Fig.3 it is clear to see that $\rho\left(\sigma^{i s o}, \alpha=-0.2\right)$ (solid line) is larger than $\rho\left(\sigma^{\text {noiso }}, \alpha=-0.2\right)$ (dot line) and $\rho\left(\sigma^{i s o}, \alpha=0.0\right)$ (dashed line)is larger than $\rho\left(\sigma^{\text {noiso }}, \alpha=0.0\right)$ (dot-dashed line) because the larger collision number from $\sigma^{i s o}$ increases the nuclear stopping and dissipation, which enhances the nuclear density, compared to the case with $\sigma^{\text {noiso }}$. From Fig.3 we can also see that $\frac{\rho}{\rho_{0}}$ decrease quickly with increasing the time after peak of $\frac{\rho}{\rho_{0}}$. Because the larger compression produces quick expanding process of the colliding system , while the small compression induces slow expanding process, at the same time, the $\frac{\rho}{\rho_{0}}$ decrease quickly with expanding process of system. But the decreasing velocity of $\frac{\rho}{\rho_{0}}$ is larger for the quick expansion system than that for the slow expansion system, up to about after $70 \mathrm{fm} / \mathrm{c}$, on the contrary, $\rho\left(\sigma^{\text {noiso }}, \alpha=-0.2\right)$ (dot line) is larger than $\rho\left(\sigma^{i s o}, \alpha=-0.2\right)$ (solid line) and $\rho\left(\sigma^{\text {noiso }}, \alpha=0.0\right)$ (dot-dashed line) is larger than $\rho\left(\sigma^{i s o}, \alpha=0.0\right)$ (dashed line). In particular, the gap between two lines for $\alpha=-0.2$ is larger than that for $\alpha=0.0$ after about $70 \mathrm{fm} / \mathrm{c}$. This property is very similar to the $N_{i m f}$, which means that the medium correction of an isospin dependent nucleon nucleon cross section enhances also the dependence of $\rho$ on the isospin effect of two-body collision,including the role of MDI on it, which induces the same effects to $N_{i m f}$ through the nucleon nucleon cross section as a function of the nuclear density as shown in Eg.(5).

In summary,the calculation results by using IQMD show that (1) the multiplicity of intermediate mass fragments $N_{i m f}$ with $\sigma^{i s o}$ are always larger than those with $\sigma^{\text {noiso }}$ in the energy region studied here. (2) In particular,the difference between the two values of $N_{i m f}$ from the $\sigma^{i s o}$ and $\sigma^{\text {noiso }}$ for $\alpha=-0.2$ is larger than corresponding that for $\alpha=0.0$, i.e., the medium correction of the isospin dependent nucleon nucleon cross section enhances the dependence of $N_{i m f}$ on the isospin effect of isospin dependent nucleon nucleon cross section . (3) MDI produces an important role for 
enhancing the isospin effect of two-body collision from the medium correction on the $N_{i m f}$.

\section{ACKNOWLEDGMENT}

We thank Prof.Bao-An Li for helpful discussions.

This work is supported by the Major State Basic Research Development Program in China Under Contract No.G2000077400, "100-person project" of the Chinese Academy of Sciences, the National Natural Science Foundation of China under Grants Nos.10175080, 10004012,10175082 and The CAS Knowledge Innovation Project No. KJCX2-SW-N02

\section{References}

[1] Bao-An Li and W. Udo Schröder,Isospin Physics in Heavy-Ion Collisions at Intermediate Energies (book), Nova Science Publishers, Inc (2001, New York).

[2] Bao-An Li, C.M. Ko and W. Bauer, Int. J. Mod. Phys. E7,No.2,147 (1998).

[3] M.S. Hussein R.A. Rego and C.A. Bertulani, Phys. Rep. 201,279(1993).

[4] H.A. Bethe, ReV. Mod. Phys.62,801 (1990).

[5] C.J. Pethick and D.G. Ravenhall, Ann. Rev. Nucl. Part. Sci.45,429 (1995).

[6] R. Wada et al., Phys. Rev. Lett. 58,1829 (1987).

[7] S.J. Yennello et al., Phys. Lett. B321,14 (1994) Nucl.Phys.A681,317c (2001) and referces therein.

[8] R. Pak et al., Phys. Rev. Lett. 78,1022 (1997); 78, 1026 (1997).

[9] G.D. Westfall, Nucl. Phys. A630,27c (1998); A681,343C (2001).

[10] G.J. Kunde et al., Phys. Rev. Lett. 77,2897 (1996).

[11] M.L. Miller et al., Phys. Rev. Lett. 82,1399 (1999). 
[12] H. Xu et al., Phys. Rev. Lett. 85, 16(2000); M.B.Tsang et al., Phys. Rev. Lett. 86,5023 (2001).

[13] W. Udo Schröder et al., Nucl. Phys. A681,418c (2001); and references therein.

[14] L.G. Sobotka et al., Phys. Rev. C55,R1272 (1994); 62, 031603 (2000).

[15] F. Rami et al., Phys. Rev. Lett. 84,1120 (2000).

[16] W.P. Tan et al., Phys. Rev.C64,R051901 (2001).

[17] M. Farine, T. Sami, B. Remaud and F. Sebille, Z. Phys. 339, 363 (1991).

[18] H. Müller and B.D. Serot, Phys. Rev. C52, 2072 (1995).

[19] Bao-An Li et al., Phys. Rev. Lett. 76, 4492 (1996); 78, 1644 (1997); 85, 4221 (2000).

[20] G. Kortmeyer, W. Bauer abd G.J. Kunde, Phys. Rev. C55, 2730 (1997).

[21] M. Colonna et al., Phys. Lett. B428, 1(1998); V. Baran et al., Nucl., Phys. A632, 287 (1998). M.Di Toro et al. Nucl. Phys. A681,426c(2001) and references therein.

[22] J. Pan and S. Das Gupta, Phys. Rev. C57, 1839 (1998).

[23] Ph. Chomaz and F. Gulminelli, Phys. Lett. B447, 221 (1999).

[24] A. Hombach et al., Eur. Phys. J. A5, 77 (1999).

[25] Jian-Ye.Liu, Qiag Zhao, Shun-Jin Wang, Wei Zuo and Wen-Jun Guo, Nucl. Phys. A687, 475 (2001).

[26] D. Klakow, G. Welke and W. Bauer, Phys. Rev. C48, 1982 (1993).

[27] K.Chen, Z. Fraenkel et al., Phys. Rev. 166, 949 (1968).

[28] J. Aichelin, Phys. Rep. 202, 233 (1991); Ch. Hartnack et al., Euro. J. Nucl. Phys. A1, 151 (1998).

[29] Bao-An Li, Nucl. Phys. A681, 434c (2001).

[30] J. Aichelin et al., Phys. Rev. Lett. 58, 1926 (1987). 
[31] J. Aichelin et al., Phys. Rev. C37, 2451 (1988).

[32] P.G. Reinhard et al., Computational Nuclear Physics,Vol.1, Springer-Verlag, Berlin,1991,pp.28-50 Danielewicz, Nucl. Phys. A673, 375(2000).

[33] G. Alkahzov et al., Nucl. Phys. A280, 365 (1977).

[34] G.F. Bertsch and S.D. Gupta, Phys.Rep. 160,1991 (1988).

[35] Jian-Ye Liu, Wen-Jun Guo, Shun-Jin Wang, Wei Zuo,Qiang Zhao and YanFang Yang, Phys. Rev. Lett. Vol. 86, No.6,975 (2001) ,Yan-Fang Yang,JianYe Liu,Wei Zuo, Wen-Jun Guo and Qiang Zhao, Chin. Phys. Lett. Vol. 18, No.8,1040 (2001).

[36] Jian-Ye Liu, Qiang Zhao, Shun-Jin Wang, Wei Zuo and Wen-Jun Guo, Phys.Rev.C63,054612(2001).

[37] Jian-Ye Liu,Yan-Fang Yang and Wen-Jun Guo, Yong-Zhong Xing, Wei Zuo, Xi-Guo Lee, Zeng-Hua Li, Phys. Lett.B540,No.3-4,213 (2002).

[38] Jian-Ye Liu, Yong-Zhong Xing and Wen-Jun Guo Chin. Phys. Lett. Vol. , No. , (2003) in press.

\section{Figure captions}

Fig.1 An impact parameter average value of the multiplicity of intermediate mass fragments, $<N_{i m f}>_{b}$ as a function of the beam energy for $U_{0}^{\text {sym }}, U_{1}^{\text {sym }}$ and $U_{2}^{\text {sym }}$ with $\sigma^{\text {iso }}$ and $\sigma^{\text {noiso }}$ for the mass symmetry system ${ }^{76} \mathrm{Kr}+{ }^{76} \mathrm{Kr}$ and asymmetry mass system ${ }^{112} \mathrm{Sn}+{ }^{40} \mathrm{Ca}$ (see text).

Fig.2 The time evolution of the $N_{i m f}$ with MDI and NOMDI for four cases (see text) and systems ${ }^{76} \mathrm{Kr}+{ }^{76} \mathrm{Kr}$ at $\mathrm{E}=150 \mathrm{MeV} /$ nucleon and $\mathrm{b}=4.0 \mathrm{fm}$.

Fig.3 The time evolution of the ratio of nuclear density to normal density $\frac{\rho\left(\sigma^{i s o}, \alpha\right)}{\rho_{0}}$ for different cases(see text). 